\title{
Ethnographies politiques de la violence
}

Introduction

\section{Chiara Calzolaio, Pamela Colombo et Chowra Makaremi}

\section{(2) OpenEdition Journals}

Édition électronique

URL : http://journals.openedition.org/conflits/19335

DOI : $10.4000 /$ conflits. 19335

ISSN : $1777-5345$

Éditeur :

CCLS - Centre d'études sur les conflits lilberté et sécurité, L'Harmattan

Édition imprimée

Date de publication : 20 décembre 2016

Pagination : 7-13

ISBN : 978-2-343-11087-5

ISSN : 1157-996X

Référence électronique

Chiara Calzolaio, Pamela Colombo et Chowra Makaremi, «Ethnographies politiques de la violence », Cultures \& Conflits [En ligne], 103-104 | automne/hiver 2016, mis en ligne le 20 décembre 2016 consulté le 30 mars 2021. URL : http://journals.openedition.org/conflits/19335 ; DOI : https://doi.org/ 10.4000/conflits.19335 


\section{Ethnographies politiques de la violence}

Introduction

\section{Chiara CALZOLAIO, Pamela COLOMBO et Chowra MAKAREMI}

Chiara Calzolaio est doctorante en anthropologie sociale et ethnologie à l'EHESS. Elle s'intéresse aux manifestations contemporaines de la violence, notamment aux violences de genre et à celles liées à la criminalité organisée et à la lutte contre le narcotrafic an Mexique, en conjuguant deux niveaux d'analyse : celui des expériences corporelles, subjectives et sociales de la violence et celui de sa problématisation publique et politique.

Pamela Colombo est docteure en sociologie de l'Université du Pays Basque. Cherchense Marie Sktodowska-Curie à l'EHESS et associée au programme ERC "Corpses of Mass Violence and Genocide » (2014-2016), elle travaille actuellement sur la construction des "villages stratégiques » en Amérique latine. Ses publications portent principalement sur le lien entre espace et violence d'État.

Chowra Makaremi est chargée de recherche an CNRS, membre de l'Institut de Recherche Interdisciplinaire sur les Enjeux Sociaux (IRIS). Ses travaux portent sur l'anthropologie de l'État, les formes juridiques et ordinaires de la violence et l'expérience qu'en font les sujets, notamment en situation d'exil.

T a définition de la violence politique a donné lieu à des débats dans les Lsciences politiques comme en anthropologie ${ }^{1}$. Elle recouvre un vaste spectre de situations (arrestations, détentions extra-légales, tortures, disparitions forcées, exécutions, tueries, massacres, guerres civiles, opérations de contre-insurrection...) qui peuvent se superposer dans les faits, et dont la qualification - divergeant au gré des acteurs et des mémoires - est elle-même un enjeu politique. Ce dossier réunit une série d'ethnographies qui, en évitant une définition a priori de la violence, préfèrent diriger le regard vers les expériences vécues qui en sont faites, et les rapports sociaux dans lesquels elles s'in-

1. Voir Nagengast C., "Violence, Terror, and The Crisis of The State ", Annual Review of Anthropology, vol. 23, n 1, 1994, pp. 109-136; Bourgois P., « La violence en temps de guerre et en temps de paix. Leçons de l'après-guerre froide : l'exemple du Salvador ", Cultures $\mathcal{E}$ Conflits, $\mathrm{n}^{\circ}$ 47, 2002, pp. 81-116. 
sèrent. Par ethnographie, nous entendons une modalité d'appréhension du réel, mais aussi une pratique réflexive sur ses pratiques empiriques de recherche (qui ne se limitent pas à faire des observations et des entretiens, ou les deux), sur ses expressions théoriques et ses pratiques d'écriture. Fondées sur des terrains approfondis, ces ethnographies sont politiques en ce qu'elles permettent de penser la violence dans son rapport au conflit et au pouvoir, que ce soit du point de vue de son maintien ou de sa contestation.

L'anthropologie n'est certes pas la discipline qui s'est intéressée le plus tôt et le plus volontiers à la question de la violence politique. Au contraire, le prisme du relativisme culturel quant à ce qui est « violent » et le refus d'aborder les enjeux politiques contemporains de la recherche - du fait d'une complicité directe avec l'entreprise coloniale, puis plus indirecte avec la domination occidentale ${ }^{2}$, a amené les anthropologues - y compris les plus prestigieux - à se taire de façon notoire sur des terrains de violence. Par exemple Julian Pitt-Rivers peut étudier les hiérarchies politiques et sociales d'une communauté rurale espagnole en 1954, en laissant entièrement de côté le franquisme et son mode brutal de (re)production des rapports sociaux. Clifford Geertz parvient quant à lui à écrire son étude de référence sur les combats de coq en Indonésie sans mentionner une seule fois le massacre des communistes qui vient d'avoir lieu sur son terrain ${ }^{3}$. Toutefois, après la guerre froide, la discipline commence à s'intéresser à la question de la violence et réfléchit sur les enjeux de terrains « sous les balles », ou « en état d'urgence » ${ }^{4}$, ouvrant la voie, à partir des années 2000 , à une anthropologie politique de la violence fondée sur un travail ethnographique approfondi ${ }^{5}$. Longtemps absente en France ${ }^{6}$, la question a été rouverte à la faveur de cette littérature récente 7 . Ce numéro de Cultures E Conflits s'inscrit dans cette continuité, explorant ce que cette production scientifique contemporaine, moins diffusée en France, apporte aux études de la violence dans son rapport au pouvoir, à l’État, et au conflit. Il pro-

2. Ces liens (et conditions de production de la connaissance) sont notamment analysés dans Geertz C. et G. Marcus, Writing Culture. The Poetics and Politics of Ethnography, Berkeley, University of California Press, 1986.

3. Pitt Rivers J., The people of the Sierra, Chicago, University of Chicago Press, 1971 (1954); Clifford G., " Deep Play: Notes on the Balinese Cockfight ", Daedalus, vol. 101, n 1, «Myth, Symbol, and Culture », hiver 1972, pp. 56-86.

4. Voir Nordstrom C. et A. Robben, «Introduction », et Feldman A., «Ethnographic States of Emergency », in Nordstrom C. et A. Robben (eds.), A. Fieldwork Under Fire. Contemporary Studies of Violence and Survival, Berkeley, University of California Press, 1995, pp. 1-23 et pp. 224-252.

5. Ce champ a notamment été balisé par la trilogie : Kleinman A., Das V. et M. Lock (eds.), Social Suffering, Berkeley, University of California Press, 1997 [1996] ; Das V., Kleinman A., Ramphele M. et P. Reynolds (eds.), Violence and Subjectivity, Berkeley, University of California Press, 2000 ; Das V., Kleinman A., Lock M., Ramphele M. et P. Reynolds (eds.), Remaking a World: Violence, Social Suffering, and Recovery, Berkeley, University of California Press, 2001. Voir aussi : Scheper-Hughes N. et P. Bourgois (eds.), Violence in War and Peace: An Anthology, Malden, MA, Blackwell, 2003 ; Fassin D., "The Trace: Violence, Truth, and the Politics of the Body », Social Research, vol. 78, n 2, 2011, pp. 281-298.

6. À de rares exceptions près comme Lenclud, G., Claverie E. et J. Jamin, « Une ethnographie de la violence est-elle possible ? » Études rurales, n 95-96, 1984, pp. 9-21. 
longe une série de réflexions de la revue sur les usages de la violence dans les démocraties et les pratiques illibérales qu'elles déploient sur ce terrain ${ }^{8}$.

Tout en prenant appui sur un vaste travail de théorisation de la violence qui a accompagné le $\mathrm{XX}^{\mathrm{e}}$ siècle 9 , les ethnographies politiques qui composent ce numéro cherchent à se détacher d'un biais qui a longtemps marqué les sciences sociales : celui de la dichotomie entre, d'un côté, l'intellect, le discours, l'idéologie et de l'autre, le corps, la violence physique et le domaine de l'irrationnel. Elles proposent ainsi de se détacher d'une approche essentialiste, pour aborder les situations de violence à l'échelle des expériences individuelles et collectives. La violence politique peut être contestataire et revendiquée comme outil d'opposition à l'ordre social et politique. Mais elle est aussi, et souvent, organisée par l'État (et/ou les élites) et légitimée par le maintien de l'ordre. Ce dossier propose d'explorer les relations qu'entretiennent ces deux dynamiques et leurs effets sur les subjectivités des individus. Si les ethnographies politiques de la violence se distinguent par le brouillage des dichotomies, c'est aussi en refusant celle entre terrains lointains, exotiques, où prévaut encore une lecture essentialiste, et un usage pléthorique du concept de culture comme prisme d'interprétation et d'explication, et les terrains proches, c'est-à-dire les sociétés occidentales où la question de la violence est presque exclusivement étudiée comme processus politique, souvent saisi dans des débats juridiques. Or, se déprendre de la distinction entre « eux » et «nous » permet d'affranchir l'analyse des démarcations normatives et des catégorisations qui définissent les différents régimes politiques : démocraties, États de droit, dictatures, États faillis, etc.

En ouverture du numéro, Chowra Makaremi introduit les débats théoriques et méthodologiques, mais aussi les enjeux épistémiques qui traversent la littérature anthropologique contemporaine. Chiara Calzolaio explore, dans leur expérience mais aussi leur narration, les pratiques de la violence d'État qui ont accompagné les opérations militaires de lutte contre le narcotrafic au Mexique. Andrew Crosby et Andrea Rea se penchent quant à eux sur les

7. Entre autres : Naepels M., «Quatre questions sur la violence », L'Homme, vol. 177-178, 2006, pp. 487-495 et Conjurer la guerre : violence et pouvoir à Honaïlou, Nouvelle-Calédonie, Paris, France, École des hautes études en sciences sociales, 2012 ; Lavergne C. et A. Perdoncin, «La violence à l'épreuve de la description », Tracés. Revue de Sciences Sociales, "Décrire la violence ", n 19, 2010, pp. 5-25 ; Bourgois P., " Théoriser la violence en Amérique », L'Homme, 2012, vol. 203-204, pp. 139-168.

8. Voir notamment « La violence dans les démocraties occidentales $»\left(n^{\circ} 9-10,1993\right)$, « Les disparitions » $\left(n^{\circ} 13-14,1994\right)$, «Construire l'ennemi intérieur » $\left(n^{\circ} 43,2001\right)$, "Suspicion et exception » $\left(\mathrm{n}^{\circ} 58,2005\right)$, «Antiterrorisme et société » $\left(\mathrm{n}^{\circ} 61,2006\right)$, « Le passage par la violence en politique » ( $\left.n^{\circ} 81-82,2011\right)$, « Militantisme et répression » $\left(n^{\circ} 89,2013\right)$.

9. Arendt H., The origins of totalitarism, New York, Brace and Co, 1951 ; Benjamin W., «Critique of violence », Reflections, vol. 14, n 3, 1978 (1927), pp. 277-300; Canetti E., Masse et puissance, Paris, Gallimard, 1966 ; Foucault M., Sécurité, territoire, population. Cours an Collège de France, 1977-1978, Paris, Gallimard, 2004 ; Tilly C., The politics of collective violence, Cambridge, Cambridge University Press, 2003 ; Weil S., "L'Iliade ou le poème de la force », in Euvres, Paris, Gallimard, coll. «Quarto », 1999, pp. 548-49. 
dynamiques de production de sujets « indésirables » en analysant les pratiques de contrôle policier dans un aéroport européen et les violences symboliques aussi bien que les espaces de pouvoir discrétionnaire qu'elles engendrent. Pamela Colombo étudie la vie quotidienne au sein des «villages stratégiques » - dispositifs spatiaux de contre-insurrection créés pendant la dictature militaire en Argentine. Sabrina Melenotte aborde les discours, qualifications et assignations produits autours du massacre d'Acteal comme une voie d'accès à la compréhension du phénomène paramilitaire et, plus largement, du fonctionnement du régime mexicain. Kimberly Theidon, enfin, explore les usages politiques des récits de la guerre civile dans une région rurale du Pérou et notamment la manière dont ils participent à la création de nouveaux rapports de pouvoir, d'ethnicité et de genre. Nous avons jugé utile d'introduire ce travail, inédit en français, qui présente les modalités théoriques et méthodologiques d'une anthropologie de la violence à la lumière des enjeux de genre et montre toute la pertinence et la richesse conceptuelle de cette grille d'analyse.

Résolument pluridisciplinaires, les articles réunis dans ce numéro présentent un éventail de la diversité méthodologique qui caractérise le travail ethnographique. En reprenant les théories de la «street-level bureaucracy", Andrea Rea et Andrew Crosby analysent ainsi la mise en œuvre des politiques publiques de contrôle des frontières européennes à travers une observation des pratiques ordinaires et des interactions entre gardes-frontières et voyageurs. En se basant sur des récits de vie et des récits de soi de victimes non organisées en association, Chiara Calzolaio creuse les effets des narrations hégémoniques de la violence sur les expériences sociales. Or, la pratique ethnographique ne se limite pas à la présence sur le terrain, mais peut s'ouvrir également à des façons de travailler avec des documents écrits, des dispositifs d'écritures saisis comme tels et éclairés en retour par l'observation. Sabrina Melenotte travaille ainsi à partir de la production existante d'une littérature juridique autour de la qualification d'un massacre et de ses acteurs. À partir de recherches de terrains approfondies, Pamela Colombo et Kimberly Theidon abordent les expériences des paysans qui ont subi la mise en place de différentes politiques de contre-insurrection en Argentine et au Pérou en les mettant en dialogue avec l'analyse de textes écrits, qu'il s'agisse de la documentation militaire ou des Libros de Actas des communautés indiennes. Cette démarche permet d'approcher la complexité des mémoires et le rôle qu'y jouent les différentes productions écrites. Chowra Makaremi discute la question de la présence sur le terrain et la possibilité d'une ethnographie de l'aprèscoup ou «à distance ». Dans cette diversité d'approches, toutefois, une exigence partagée par toutes les contributions demeure la prise en compte de ce que Kimberly Theidon nomme la «polyphonie ». Il s'agit en effet de laisser leur place au foisonnement des voix et aux contradictions qui font l'étoffe de la réalité vécue, sans opération de lissage ou point de vue surplombant unificateur. 
L'attention portée aux expériences vécues permet d'approcher la violence politique dans son quotidien, que ce soit dans les pratiques routinières du travail des agents de l'État dans un aéroport européen, dans l'organisation de la vie dans un «village stratégique » sous la dictature militaire en Argentine ou dans une ville frontalière du Mexique dans les années de la guerre contre la drogue. Ces observations du routinier, y compris en situation d'exception, permettent d'entrer dans le grain fin des situations de violence. Mais elles permettent aussi, à travers une opération de montée en généralité, d'en compléter, d'en renouveler, d'en infléchir l'analyse conceptuelle et théorique. Notamment, les études du quotidien explorent les mécanismes politiques et sociaux à travers lesquels la violence est produite et produit, à son tour, des régimes de vie, des espaces, des sujets.

En explorant terrains proches et lointains, systèmes politiques dictatoriaux ou démocratiques, les ethnographies présentées dans ce numéro analysent la complexité des régimes de pouvoir au-delà ou en-deçà des catégories instituées de la science politique. Andrea Rea et Andrew Crosby s'attachent au déploiement du pouvoir discrétionnaire aux frontières des États de droit européens dans la gestion policière des mobilités. Chiara Calzolaio étudie le traitement des délinquants comme "ennemis » dans une guerre contre la drogue qui implique un continum complexe et opaque d'acteurs policiers et militaires au Mexique. À l'inverse, Pamela Colombo observe les politiques "d'action civique » des militaires argentins, analysant l'inclusion forcée de paysans subalternes dans une nouvelle citoyenneté façonnée à travers les villages stratégiques - villages situés à quelques kilomètres seulement des centres de détention et de torture où disparaissaient d'autres citoyen(ne)s considérés comme « subversifs ». L'ethnographie de l'État à l'épreuve de la violence met ainsi en lumière la porosité des frontières entre ce qui est étatique et ce qui ne l'est pas, tout en ouvrant des pistes de réflexions autour des circulations des savoirs et des pratiques sécuritaires - que ce soit à travers la réactivation d'héritages ou les transferts transnationaux.

Cette approche pose la question de la visibilité et de l'invisibilité de la violence - de son invisibilisation et de sa visibilisation, tant par les dispositifs du pouvoir que par ceux de l'écriture. Si le massacre d'Acteal dans le Chiapas ou le conflit armé entre Sentier lumineux, militaires et paramilitaires au Pérou sont des situations de violence collective évidente, d'autres terrains présentés dans ce numéro nous confrontent à la question de la violence là où elle ne se pose pas de manière aussi directe dans ses effets, tel que les villages stratégiques en Argentine ou les postes de contrôle aéroportuaires aux frontières européennes. Si le terrain ethnographique rend visible ce qui ne l'était pas, il peut aussi changer notre lecture de ce qui l'était déjà, ce que l'on observe de prime abord, ou la documentation écrite avec laquelle on travaille. Ce travail 
ouvre des pistes de recherche sur le fonctionnement du pouvoir, sa localisation, ses productions.

L'une de ces dimensions est celle des relations entre espaces et violences. Les dispositifs spatiaux de contrôle, tels que les aéroports ou les villages stratégiques, sont produits par des régimes de gouvernementalité différents, dont l'étude nous permet de saisir comment le pouvoir se spatialise et circule dans la société. Un enjeu de la violence renvoie alors aux façons dont l'État investit et se rend présent dans des espaces contestés, des territoires marginaux - les zones rurales isolées, des régions travaillées par des processus d'autonomisation politique, des zones franches, l'espace ambigu des aéroports, à la fois zones internationales et frontières d'États - pour y produire des citoyens ou des non-citoyens.

Les rapports entre violence et subjectivité sont abordés sous plusieurs angles. Certaines analyses s’intéressent aux modalités par lesquelles la violence façonne des sujets politiques marginaux : les usagers de drogue (victimes invisibilisées de la lutte contre le narcotrafic au Mexique) ou les voyageurs irréguliers incarcérés et renvoyés dans les espaces de frontière. D’autres creusent la question des acteurs de la violence, les manières dont l'État les «fabrique ", s'appuie sur eux, les soutient et, à travers ce processus, s'enracine (au Pérou andin) et se perpétue dans la longue durée (en Argentine). Si les ethnographies analysent la production de formes d'appartenance nationales dans les zones grises de la violence étatique, elle mettent en lumière comment ces formes de (non)citoyenneté se construisent dans les faits au contact d'une violence d'État qui se légitime et s'appuie sur des figures de l'ennemi : le migrant clandestin, le subversif, le narcotraficant, le terroriste, le guérillero.

Par ailleurs, la production des sujets politiques considérée au prisme de l'appartenance et des identités collectives pose la question de la mémoire, ou plutôt des mémoires de la violence. Le travail de terrain ne donne pas accès à une « réalité historique » des événements, mais permet d'en explorer les expériences vécues et leur impact dans un temps long. Plusieurs textes montrent comment des cadres interprétatifs hégémoniques sont élaborés ou repris par différents acteurs. Kimberly Theidon montre ainsi comment, au Pérou, les milices paysannes, les «ronderos ", s'approprient la notion de citoyenneté en reprenant à leur compte les normes de genre qui survalorisent une masculinité guerrière, héritées de la formation militaire à travers laquelle l'État s'est rendu présent dans ces territoires relégués. Le droit apparaît également comme un outil de production et un espace polémique de mémoire collective. C'est ce qu'illustre Sabrina Menelotte à travers le traitement juridique d'un massacre par l'État mexicain, ou encore Chowra Makaremi avec l'évocation des « tribunaux d'opinion » qui utilisent les fonctions symboliques du droit pour 
dénoncer et pallier l'impunité des crimes d'État, mais aussi les documenter en fabriquer une contre-mémoire.

Or, la question de la violence en tant qu'elle est saisie par le droit introduit la figure de victime, qui est d'abord une catégorie juridique. Observer les relations entre violences et qualifications juridiques de la violence permet de montrer les hiérarchisations implicites à l'intérieur de cette catégorie, entre par exemple les victime «légitimes » et les victimes «illégitimes » de la violence d'État au Mexique que sont les usagers de la drogue ; mais aussi les renversements possibles de « bourreau » à « victime » dans le cas de la relecture du massacre d'Acteal une décennie après les événements. Pour autant, il ne s'agit pas de simplifier les relations entre violence et droit en présentant le droit comme ce qui s'oppose à la violence - ce qui vient la contenir, la résoudre, la résorber, y substituer d'autres régimes de conflictualité. C'est ce que rappelle l'article d'Andrea Rea et Andrew Crosby, en s'attachant à la violence et aux espaces de pouvoir qu'elle produit au sein de l'État de droit.

Ainsi, en assemblant des sujets qui ne l'auraient pas été sauf à considérer les approches anthropologiques dont ils relèvent, ce numéro souhaite enrichir la question des usages, des effets et des réalités vécues de la violence, dans une analyse empiriquement fondée des rapports entre pouvoir et résistance, obéissance et consentement, mais aussi de la transformation des subjectivités politiques. 